\title{
Analysis of cardiovascular risks for erectile dysfunction in Chinese patients with type 2 diabetes mellitus lacking clinical symptoms of cardiovascular diseases
}

\author{
Penghui Yuan ${ }^{1 \#}$, Delin Ma ${ }^{2 \#}$, Yucong Zhang ${ }^{1}$, Xintao Gao ${ }^{1}$, Jiaxin Wang ${ }^{1}$, Rui Li ${ }^{1}$, Zhuo Liu ${ }^{1}$, Tao Wang ${ }^{1}$, \\ Yan Yang ${ }^{2}$, Gang Yuan ${ }^{2}$, Xuefeng Yu ${ }^{2}$, Shaogang Wang ${ }^{1}$, Jihong Liu ${ }^{1}$, Xiaming Liu ${ }^{1}$ \\ ${ }^{1}$ Department of Urology and Institute of Urology, Tongji Hospital, Tongji Medical College, Huazhong University of Science and Technology, \\ Wuhan, China; ${ }^{2}$ Department of Endocrinology, Tongji Hospital, Tongji Medical College, Huazhong University of Science and Technology, Wuhan, \\ China \\ Contributions: (I) Conception and design: P Yuan, D Ma, X Liu; (II) Administrative support: T Wang, S Wang, J Liu; (III) Provision of study materials \\ or patients: Y Zhang, Y Yang, G Yuan, X Yu; (IV) Collection and assembly of data: X Gao, J Wang, R Li, Z Liu; (V) Data analysis and interpretation: \\ P Yuan, D Ma; (VI) Manuscript writing: All authors; (VII) Final approval of manuscript: All authors. \\ "These authors contributed equally to this work. \\ Correspondence to: Dr. Xiaming Liu; Dr. Jihong Liu. Department of Urology, Tongji Hospital, Tongji Medical College, Huazhong University of \\ Science and Technology, Wuhan 430030, China. Email: xmliu77@hust.edu.cn; jhliu@tjh.tjmu.edu.cn.
}

Background: Erectile dysfunction (ED) is common but usually underdiagnosed in diabetics. The correlation between different vascular lesions and ED in diabetics without clinical cardiovascular symptoms is unknown. The aim was to explore the association between cardiovascular risks and ED in Chinese type 2 diabetic men lacking clinical performance.

Methods: Erectile function of patients with type 2 diabetes was assessed by the 5 -item International Index of Erectile Function (IIEF-5) questionnaire. The data of clinical characteristics and vascular lesions at carotid and lower limb sites assessed by the Doppler ultrasound were collected to evaluate diabetesmetabolic indices. Univariate and multivariate analyses were conducted to find statistical correlation between cardiovascular risks and diabetic ED.

Results: A total of 71.21\% reported suffering from ED. Lower limb plaques were more common (45.38\%) than carotid district (35.62\%) in diabetes. Men with ED had higher carotid intima-media thickness (IMT) $(\mathrm{P}<0.001)$ and the presence of lower limb plaques $(\mathrm{P}<0.001)$ compared with men without $\mathrm{ED}$. After adjusting for age, diabetic duration, blood pressure (BP) and antidiabetic medication, carotid IMT greater than $0.75 \mathrm{~mm}$ $(\mathrm{P}<0.001)$ and the presence of lower limb plaques $(\mathrm{P}=0.051)$ remained associated with the presence of ED and its severity. Compared with isolated atherosclerosis at carotid or lower limb district, vascular lesions at any site and both sites were more correlative with ED presence (all $\mathrm{P}<0.001$ ).

Conclusions: The prevalence of ED is high among Chinese diabetic men. A higher carotid IMT and the presence of lower limb plaques indicate a tight correlation between peripheral atherosclerosis and diabetic ED. ED may be the only clinical association of symptomatic cardiovascular diseases (CVDs) in diabetes. It is significant to screen ED to prevent the further development of severe symptomatic CVDs.

Keywords: Erectile dysfunction (ED); diabetes; atherosclerosis

Submitted Jun 15, 2020. Accepted for publication Sep 18, 2020.

doi: $10.21037 /$ tau-20-999

View this article at: http://dx.doi.org/10.21037/tau-20-999 


\section{Introduction}

Diabetes mellitus is an important public health problem, which is characterized by lifelong treatments and severe complications. Global estimates of diabetes revealed that the number of cases will rise to 300 million by 2025 and 693 million by 2045 (1). Diabetes-related complications could result in high medical costs, poor quality of life and even death (2). As one of diabetic complications, erectile dysfunction (ED) occurs at an early age, and is threefold more frequent in diabetic men compared with non-diabetic men, which affects $35-90 \%$ men with type 2 diabetes (3). It has a negative impact on the quality of life and selfconfidence in men (4). What's more, cumulative studies showed that ED increased the risk of cardiovascular diseases (CVDs), especially atherosclerosis (5-7), which are major causes of death in diabetes (8). In fact, the corpus cavernosum, also known as the erectile organ, is part of the systemic vasculature hence ED is closely related to CVDs. ED and CVDs share the similar pathophysiology, especially endothelial dysfunction (9). It is reported that ED preceded the progression of systemic atherosclerotic diseases and could serve as a clinical tool in early diagnosis of CVDs (10). However, many patients with diabetes suffering from atherosclerotic diseases are not inclined to complain their sexual performance, leading to ignorance of sexual dysfunction in diabetics treated by endocrinologists in China (11). It is of significant importance to expound the association between ED and CVDs and screen ED for the early diagnosis of atherosclerosis in diabetics ahead of clinical symptoms.

Some studies have evaluated the association between ED and atherosclerosis in patients with known CVDs in the general population. It is reported that ED in men with vascular risk factors was the only clinical correlation of unrecognized atherosclerosis of common carotid arteries (12). Hypertensive patients with ED had higher common carotid intima-media thickness (IMT) and carotid-femoral pulse-wave velocity (13). Also, a few studies analyzed the relationship between femoral atherosclerosis and ED in patients with obvious CVDs, and obtained different results $(7,14)$. However, almost all of the studies obtained the conclusions based on men already affected by obvious symptomatic CVDs. In fact, there are diabetic men with ED lacking clinical cardiovascular symptoms and studies of systematical evaluation of the association between different peripheral vascular atherosclerosis and ED in diabetes simultaneously in the absence of symptomatic CVDs is important but limited, especially in China.

Based on these, the study is aimed to investigate the different atherosclerotic risks (increased IMT or the presence of plaques at carotid and lower limb sites) of ED in Chinese diabetes without obvious cardiovascular performance systematically and achieve better clinical prediction in atherosclerosis. We present the following article in accordance with the STROBE reporting checklist (available at http://dx.doi.org/10.21037/tau-20-99).

\section{Methods}

\section{Study participants}

Male patients with type 2 diabetes in Tongji Hospital in Hubei, China were recruited between July 2018 and May 2019. Participants included in this study were married men with type 2 diabetes for more than half a year and in a stable relationship with their spouse for the last 6 months. The exclusion criteria for the patients were: developing symptomatic CVDs including history of coronary artery and cerebrovascular diseases, congestive heart failure, peripheral arterial diseases or having the family history; having underlying hypogonadism; a history of the unstable diabetic process like ketoacidosis and recurrent hypoglycemia in the past 6 months; use of treatments related to ED; the history of operation or other diseases which may induce ED and the history of mental and psychological diseases. This study was conducted in accordance with the Declaration of Helsinki (as revised in 2013) and approved by Institutional Research Ethics Committee of Tongji Medical College, Huazhong University of Science and Technology (S1119). Informed consent was taken from all the patients before the study. Patients were later requested to complete the 5-item International Index of Erectile Function (IIEF-5) questionnaire for the diagnosis and grading of ED severity based on their sexual life within the past 6 months.

\section{Data collection}

Besides the evaluation of erectile function using the IIEF-5 questionnaire, fundamental data including age, duration of diabetes, personal history and drug history, blood pressure (BP), weight, height and circumference were collected. The results of plasma glucose, glycated hemoglobin (HbA1c) and lipid profile including total cholesterol (TC), high-density lipoprotein cholesterol (HDL-C), low-density lipoprotein cholesterol (LDL-C) and triglyceride (TG) were obtained 
from blood sample examinations. The profiles of peripheral arteries at carotid and lower limb sites were assessed by IMT, the presence of plaques and ankle-brachial index (ABI) using the Doppler ultrasound. ABI is calculated after measuring the systolic BP of brachial, posterior tibial and pedal arteries (15).

\section{Diagnostic criteria}

ED is classified into five severity grades: absence of ED (IIEF score 22-25), mild (score 17-21), mild-moderate (score 12-16), moderate (score 8-11) and severe ED (score 5-7) (16). Hypertension was diagnosed as systolic BP $\geq 140 \mathrm{mmHg}$ and/or diastolic $\mathrm{BP} \geq 90 \mathrm{mmHg}$, or the patients were receiving antihypertensive treatment (17). Body mass index (BMI) was calculated as weight in $\mathrm{kg} /$ (the square of height in meter) $\left(\mathrm{kg} / \mathrm{m}^{2}\right)$. Central obesity was defined as a waist circumference of $90 \mathrm{~cm}$. Dyslipidemia was defined as having one or more of the following in the field survey: TC $\geq 5.18 \mathrm{mmol} / \mathrm{L}, \mathrm{HDL}-\mathrm{C}<1.04 \mathrm{mmol} / \mathrm{L}$, LDL-C $>3.37 \mathrm{mmol} / \mathrm{L}$ or $\mathrm{TG} \geq 1.70 \mathrm{mmol} / \mathrm{L}$ (17). $\mathrm{ABI}$ is obtained by the higher value of ankle pressure (posterior tibial and pedal arteries) divided by brachial artery. $\mathrm{ABI}<0.9$ is regarded as the abnormal result (18).

\section{Statistical analysis}

Continuous variables were expressed as mean and standard deviation while categorical variables as percentages. For continuous variables, normally distributed data by twosample $t$-test and non-normally distributed data by the Mann-Whitney $U$ test were conducted to examine the differences between the ED and non-ED groups. Categorical variables were analyzed using the Chi-square test. The stepwise logistic regression analysis was conducted to assess the correlation between atherosclerotic risks and ED. Receiver-operating characteristic (ROC) analysis was performed to evaluate these correlations. The odds ratio $(\mathrm{OR})$ and $95 \%$ confidence intervals were calculated. $\mathrm{P}<0.05$ was regarded as statistical significance. Statistical tests were performed by using Statistical Package for Social Science (SPSS 23.0) and GraphPad Prism 6.0.

\section{Results}

A total of 330 patients were recruited into this study. After excluding 66 patients according to the criteria above, 264 patients were enrolled and analyzed further. Of the enrolled participants, 188 patients $(71.21 \%)$ had ED while 76 patients $(28.79 \%)$ were without ED. The characteristics of the study population were presented in Table 1 . The mean age was $48.28 \pm 7.03$ years and the mean duration of diabetes was $6.03 \pm 5.36$ years. The mean BMI was $24.74 \pm 4.34 \mathrm{~kg} / \mathrm{m}^{2}$ and the mean value of waist circumference was $92.65 \pm 10.54 \mathrm{~cm}$, which meant participants were centrally obese overall.

Compared with the non-ED group, patients in the ED group were older $(49.85 \pm 6.25$ vs. $44.40 \pm 7.36$ years, $\mathrm{P}<0.001)$ and had a longer duration of diabetes $(7.33 \pm 5.69$ vs. $2.82 \pm 2.33$ years, $\mathrm{P}<0.001$ ) (Table 1). In addition, the $\mathrm{ED}$ group had higher levels of systolic BP $(127.59 \pm 19.49 v s$. $121.86 \pm 15.07 \mathrm{mmHg}, \mathrm{P}=0.041)$ and lower levels of $\mathrm{TC}$ (4.17 \pm 1.12 vs. $4.64 \pm 1.30 \mathrm{mmol} / \mathrm{L}, \mathrm{P}=0.011)$. These was no significant difference in $\mathrm{HbA1c}, \mathrm{BMI}$, waist circumference and the presence of dyslipidemia between the groups. Patients in the ED group presented with more frequent use of sulfonylureas (SUs) (45.74\% vs. $30.26 \%, \mathrm{P}=0.021)$ and $\alpha$-glucosidase inhibitors (AGIs) $(55.85 \%$ vs. $40.79 \%$, $\mathrm{P}=0.027)$ for antidiabetic medication.

Carotid and lower limb artery lesions in diabetics between the two groups were shown in Figure 1. Plaques were more frequent at lower limb district $(45.38 \%)$ than carotid district $(35.62 \%)$ in diabetes (Figure 1A). For carotid site, patients in the ED group had higher carotid IMT $(0.82 \pm 0.22$ vs. $0.73 \pm 0.18 \mathrm{~mm}, \mathrm{P}<0.001)$ (Figure $1 B)$. However, the presence of carotid plaques was not significantly different $(37.06 \%$ vs. $31.75 \%, \mathrm{P}=0.452)$ (Figure 1C). For lower limb site, atherosclerotic plaques were more common in men with $\mathrm{ED}(52.30 \%$ vs. $26.56 \%$, $\mathrm{P}<0.001$ ) (Figure $1 D$ ). Due to only $1.72 \%$ of the study population had an ABI lower than 0.9, we did not find the obvious difference of abnormal $\mathrm{ABI}$ within the groups (1.79\% vs. $1.56 \%, \mathrm{P}=1.000$ ) (Figure $1 E$ ).

Variables with significant difference mentioned above as well as the presence of ED were involved in stepwise logistic regression analysis. Then age, duration of diabetes, systolic $\mathrm{BP}$, the presence of carotid IMT $>0.75 \mathrm{~mm}$ and lower limb plaques were retained in the final regression model (Table 2). The results showed that age $(\mathrm{OR}=1.063, \mathrm{P}=0.027)$, duration of diabetes ( $\mathrm{OR}=1.292, \mathrm{P}<0.001)$, systolic $\mathrm{BP}(\mathrm{OR}=1.028$, $\mathrm{P}=0.017)$ and the presence of carotid IMT $>0.75 \mathrm{~mm}$ $(\mathrm{OR}=5.413, \mathrm{P}<0.001)$ were independently associated with ED. Lower limb plaques were 2.229 times more frequent in men with ED, though they were slightly dependent on other variables $(\mathrm{P}=0.051)$.

To further expound the correlation between peripheral 
Table 1 Baseline statistics of the study participants with type 2 diabetes

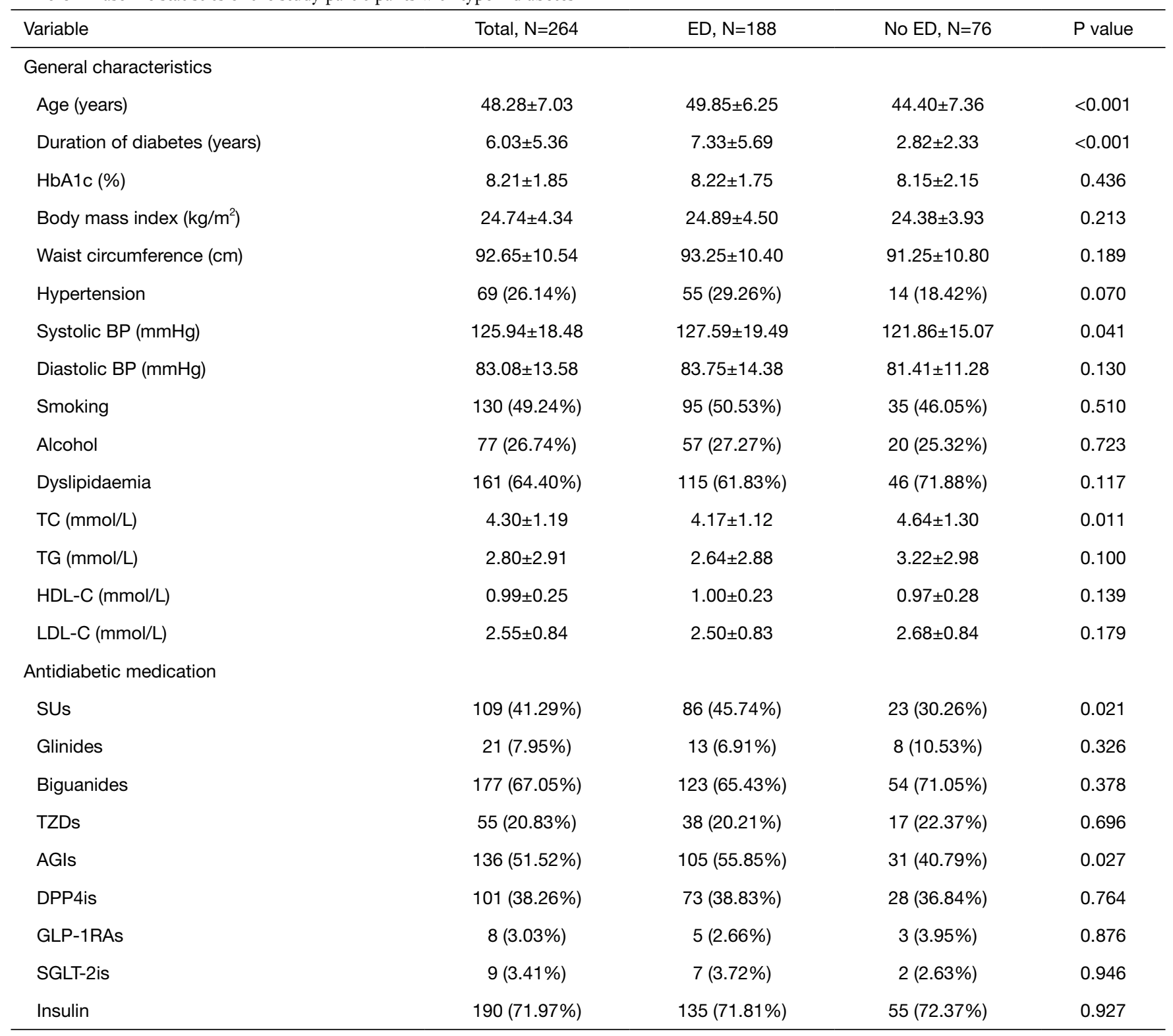

ED, erectile dysfunction; BP, blood pressure; TC, total cholesterol; TG, triglyceride; HDL-C, high-density lipoprotein cholesterol; LDL-C, low-density lipoprotein cholesterol; SUs, sulfonylureas; TZDs, thiazolidinediones; AGls, $\alpha$-glucosidase inhibitors; DPP4Is, dipeptidyl peptidase-4 (DPP4) inhibitors; GLP-1RAs, glucagon-like peptide-1 receptor agonists; SGLT-2ls, sodium-glucose cotransporter-2 inhibitors.

atherosclerosis and ED systematically, the vascular profiles were classified into vascular lesions at any site (carotid IMT $>0.75 \mathrm{~mm}$ or the presence of lower limb plaques) and at both sites (coexisting lower limb plaques and carotid IMT $>0.75 \mathrm{~mm}$ ). The correlative performance for ED and different vascular profiles including carotid IMT $>0.75 \mathrm{~mm}$, the presence of lower limb plaques, vascular lesions at any site and both sites was evaluated by ROC curves (Figure 2).
Both of carotid IMT $>0.75 \mathrm{~mm}$ and the presence of lower limb plaques had a significant correlation with $\mathrm{ED}(\mathrm{P}<0.001$ and $\mathrm{P}=0.002$, respectively), though the areas under the curve (AUC) were not high enough (0.668 for carotid IMT $>0.75 \mathrm{~mm}$ and 0.635 for the presence of lower limb plaques). However, the AUC of vascular lesions at any site (0.717, 95\% CI: $0.637-0.798, \mathrm{P}<0.001$ ) was significantly superior to that of single one. Finally, the AUC of vascular 
A

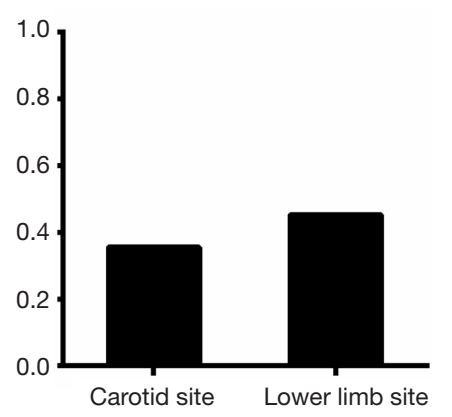

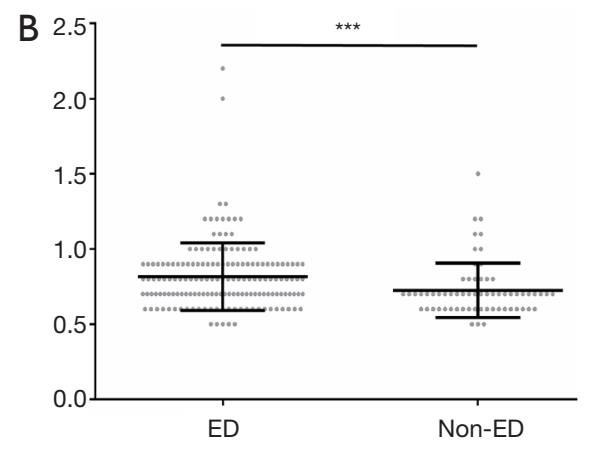

C

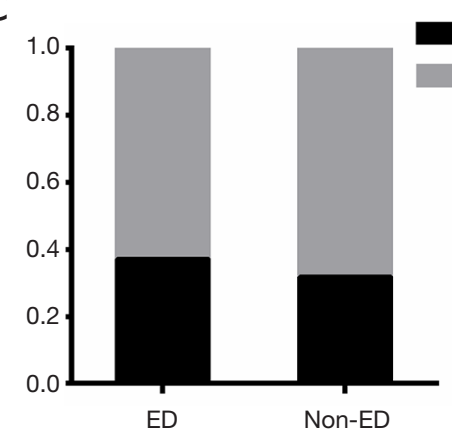

Carotid plaques $(+)$ Carotid plaques (-)

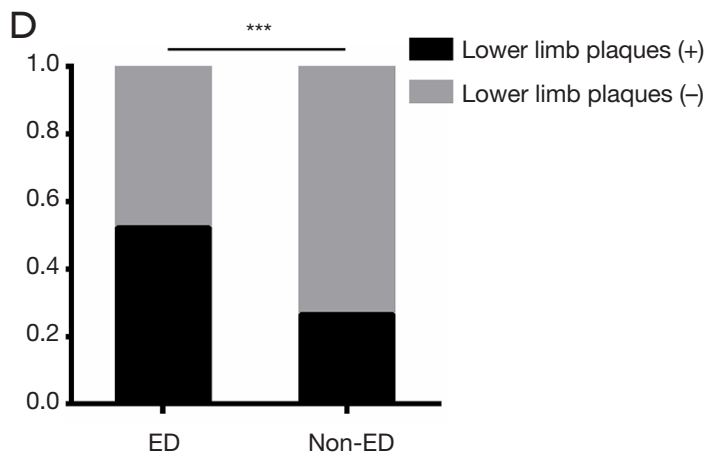

E

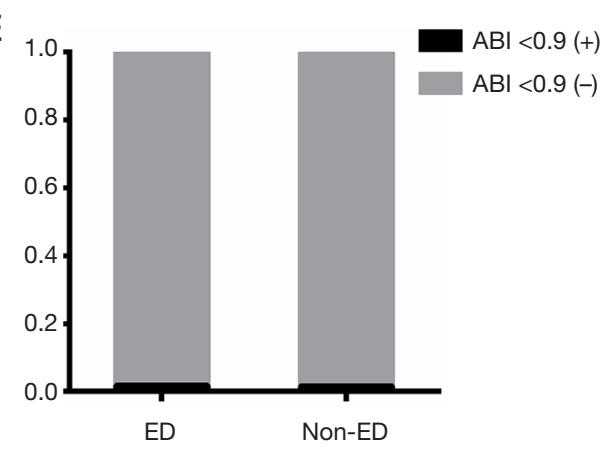

Figure 1 Peripheral atherosclerotic risk factors of the study participants with type 2 diabetes. The presence of plaques at carotid and lower limb sites in diabetes (A); carotid IMT (B), the presence of carotid plaques (C), lower limb plaques (D) and ABI $<0.9$ (E) between the ED and non-ED groups in diabetes. ${ }^{* *}, \mathrm{P}<0.05$. ED, erectile dysfunction; IMT, intima-media thickness; ABI, ankle-brachial index.

Table 2 Multivariable logistic regression analysis of risk factors for ED

\begin{tabular}{lccc}
\hline Variable & b value & Odds ratio $(95 \% \mathrm{Cl})$ & $\mathrm{P}$ value \\
\hline Age (years) & 0.061 & $1.063(1.007,1.123)$ & 0.027 \\
Duration of diabetes (years) & 0.256 & $1.292(1.148,1.453)$ & $<0.001$ \\
Systolic BP $(\mathrm{mmHg})$ & 0.028 & $1.028(1.005,1.052)$ & 0.017 \\
Carotid IMT $(>0.75 \mathrm{~mm})$ & 1.689 & $5.413(2.466,11.879)$ & $<0.001$ \\
Lower limb plaques & 0.802 & $2.229(0.999,4.979)$ & 0.051 \\
\hline
\end{tabular}

$\mathrm{ED}$, erectile dysfunction; BP, blood pressure; IMT, intima-media thickness; CI, confidence intervals. 


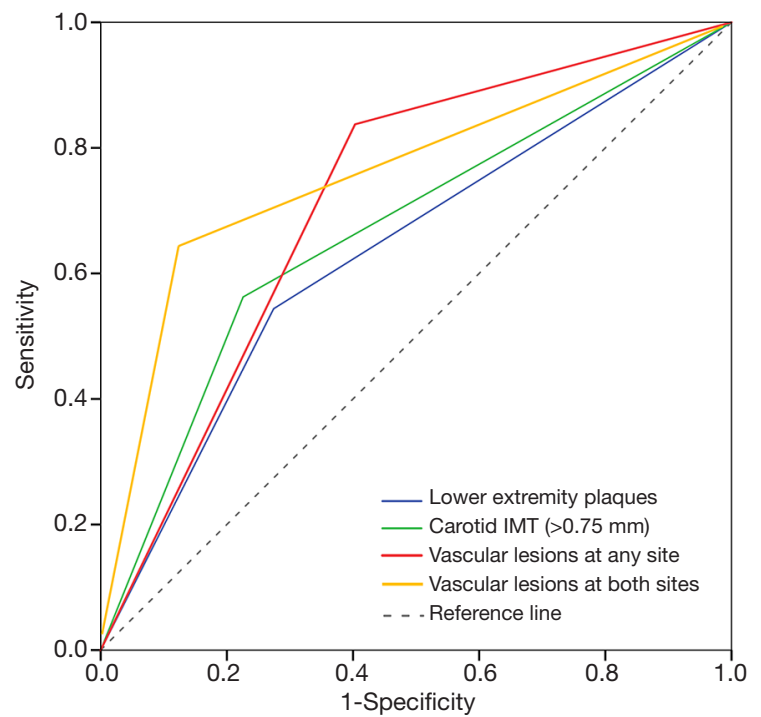

Figure 2 ROC curves for identifying the correlative performance between ED and vascular patterns. ROC, receiver-operating characteristic; ED, erectile dysfunction; IMT, intima-media thickness. lesions at both sites achieved optimal value $(0.751,95 \%$ CI: $0.658-0.843, \mathrm{P}<0.001)$, indicating the high correlation between peripheral artery lesions and ED.

Finally, the relationships between atherosclerotic lesions and the severity of ED were illustrated (Table 3). All of these four patterns increased significantly with mild, mildmoderate and moderate ED $(\mathrm{P}=0.026,<0.001$ and 0.001 for carotid IMT greater than $0.75 \mathrm{~mm}, \mathrm{P}=0.003,0.023$ and $<0.001$ for the presence of lower limb plaques, all $\mathrm{P}<0.001$ for vascular lesions at any site, and $\mathrm{P}=0.005$ and $<0.001$ for vascular lesions at both sites, respectively). Since only a relatively small number of patients developing severe ED were involved in this study, only the presence of lower limb plaques showed significant correlation with severe ED $(\mathrm{P}=0.046)$.

\section{Discussion}

ED is a common problem among men with diabetes. Though it is not a deadly disease, it significantly influences

Table 3 The association between the severity of ED and different vascular lesions

\begin{tabular}{|c|c|c|c|c|}
\hline Variable & Carotid IMT (>0.75 mm) & Lower limb plaques & Vascular lesions at any site & Vascular lesions at both sites \\
\hline OR (95\% Cl) & $2.513(1.105,5.712)$ & $3.246(1.480,7.119)$ & $5.850(2.416,14.167)$ & $7.2(1.933,26.812)$ \\
\hline$P$ value & 0.026 & 0.003 & $<0.001$ & 0.005 \\
\hline \multicolumn{5}{|c|}{ Mild-moderate ED } \\
\hline$P$ value & $<0.001$ & 0.023 & $<0.001$ & $<0.001$ \\
\hline \multicolumn{5}{|l|}{ Moderate ED } \\
\hline OR $(95 \% \mathrm{Cl})$ & $4.443(1.801,10.911)$ & $5.069(2.069,12.414)$ & $45.538(5.847,354.651)$ & $50.4(5.08,499.997)$ \\
\hline$P$ value & 0.001 & $<0.001$ & $<0.001$ & $<0.001$ \\
\hline$P$ value & 0.773 & 0.046 & 0.226 & 0.094 \\
\hline
\end{tabular}

ED, erectile dysfunction; IMT, intima-media thickness; OR, odds ratio; $\mathrm{Cl}$, confidence intervals. 
subjective well-being of life for men and his spouse and tends to be overlooked in the routine clinical screening of diabetic complications in China because of embarrassment and cultural background $(19,20)$. More importantly, ED is believed to have a tight relationship with the atherosclerotic process. There are limited studies assessing the correlation between ED and different atherosclerotic lesions systematically in Chinese diabetic population. What's more, few studies explored the research in men without clinical manifestations of CVDs. In this study, we conducted a comprehensive analysis focusing on the presence of carotid and lower limb artery lesions in Chinese diabetics with ED in the absence of cardiovascular symptoms. The prevalence of ED was $71.21 \%$ based on IIEF-5 criteria, and the incidence of ED increased with age and diabetic duration. Carotid IMT and the presence of lower limb plaques were significantly associated with ED and its severity. And the correlation achieved a better level for coexisting carotid IMT greater than $0.75 \mathrm{~mm}$ and lower limb plaques, indicating subclinical atherosclerosis requires more attention in diabetic ED.

Penile erection is described as "essentially a vascular event under neurogenic control" (21). It is reported that endothelial dysfunction impairing vasodilatation exists both in the process of ED and atherosclerosis (12). Studies showed that ED would precede the presence of clinical atherosclerosis (22) due to the fact that corpus cavernous vessels are smaller and more vulnerable to harmful conditions compared with coronary arteries and other peripheral vessels $(23,24)$. Carotid IMT shows a close relationship with CVDs (25), which could serve as a marker of subclinical atherosclerosis and atherosclerotic CVD (26). It was reported that higher carotid IMT was correlated with ED in hypertensive patients and it increased significantly with ED severity in patients with CVD risks $(7,13)$. Besides, previous study demonstrated that as carotid IMT increased, nocturnal penile erection worsened in a rigidity monitoring test (27) and patients with normal carotid IMT had a better response to PDE5Is than patients with increased carotid IMT (26). For evaluation of CVD risks, carotid IMT $\geq 1 \mathrm{~mm}$ is considered high risk $(28,29)$. In the general population of 270 patients without clinical atherosclerosis, carotid IMT greater than $1 \mathrm{~mm}$ had a 2.6 times greater risk of severe ED (12). In this study, the mean carotid IMT was $0.79 \mathrm{~mm}$ in diabetics and men with ED had significantly higher carotid IMT. The cutoff value $0.75 \mathrm{~mm}$ was obtained based on optimal sensitivity and specificity for the presence of ED. After adjusting for age, diabetic duration, BP and antidiabetic medication, carotid IMT greater than $0.75 \mathrm{~mm}$ remained independently associated with the presence of $\mathrm{ED}(\mathrm{OR}=5.413$, 95\% CI: 2.466-11.879) and its severity, suggesting more accelerated subclinical atherosclerosis in diabetic ED.

In addition to IMT, the presence of plaques is also regarded as a marker of atherosclerotic process at carotid site (30). In this study, $37.06 \%$ of diabetics with ED had carotid plaques while $31.75 \%$ in men without ED. It seemed the presence of carotid plaques was comparable between the groups, which is consistent with Lee's study (31), that's, a similar prevalence of carotid plaques in men with or without ED. However, Lahoz et al. (7) reported that the association between carotid plaques and ED remained statistically significant after adjusting for cardiovascular risk factors. In a total of 102 men with ED, plaques in the carotid artery were more frequent in men with arterial ED (32). These inconsistent results may imply discrepant profiles of IMT and plaque at carotid site. It still needs to be expounded whether the presence of carotid plaques correlates with ED.

Besides carotid lesions, peripheral atherosclerosis at lower limb site is observed in diabetes (33). In this study, lower limb plaques were more common than carotid plaques in diabetes. Whether it is related to the presence of ED in diabetes is worth exploring due to anatomical closeness with penile vessels relative to carotid arteries (34). Both of $\mathrm{ABI}$ and the presence of plaques were used for evaluation of lower limb artery lesions in this study. Of all the population, only a small part of subjects developed abnormal ABI $(<0.9)$, so the association between $\mathrm{ABI}$ and $\mathrm{ED}$ was not explored sufficiently. Similarly, in a cohort of 614 men, only $3.6 \%$ of them had ABI lower than $0.9 \mathrm{~mm}$ and statistical difference did not exist in mean ABI or the presence of abnormal ABI (7). It seemed that abnormal ABI tended to be involved in diabetics with obvious CVDs and our study included men lacking cardiovascular performance. Then we focused on plaque formation in lower limb arteries and concluded that it was more frequent in diabetics with ED. Also, Lower limb plaques were associated with different degrees of severity of ED. After adjusting for age, diabetic duration and antidiabetic medication, it remained correlative with ED, though it was slightly dependent on other variables. Besides, our study revealed that lower limb plaques were more common than those at carotid district in diabetes. There is scarce data about lower limb atherosclerosis in ED patients. In a general population, Foresta et al. (34) found ED patients presented with a significantly higher prevalence 
of femoral plaques independently from other cardiovascular risk factors. Goksu et al. (32) found 78.4\% of arterial ED patients with femoral plaques had lower level of cavernous artery peak-systolic velocity compared with patients with non-arterial ED. All of these showed femoral artery lesions shared similar pathology with vascular changes in ED and deserved consideration especially in diabetes.

To further expound the peripheral atherosclerotic profiles in diabetic ED as a whole, we evaluated the correlative performance between ED and four different vascular patterns by ROC curves. Compared with isolated abnormal carotid IMT or lower limb plaques, vascular lesions at both sites seemed more correlative with ED presence and its severity. In fact, none of these patients were aware of the presence of vascular lesions, indicating ED may be the only clinical association of symptomatic CVDs in diabetes. In a sense, penile arteries as well as other peripheral arteries like carotid and lower limb vessels are involved in systemic vasculature. For men with diabetes lacking cardiovascular symptoms, the presence of ED indicated impaired penile vascular function. In this circumstance, men had high systematic atherosclerosis risks and deserved special attention.

There were still some limitations in this study. First, this study was conducted at a large size tertiary comprehensive hospital in China where patients with more severe diabetes as well as complications were inclined to be involved. The presence of selection bias and poor representation impeded generalization of the findings to the general diabetic population. Second, this cross-sectional study limited analysis of causal associations. Third, the sample size in this study is not large enough, which may influence the evaluation between the severity of ED and different vascular patterns. Further prospective studies and animal experiments would provide more convincing results on the relationship between diabetic ED and associated atherosclerotic risks in the future.

\section{Conclusions}

In the current study, the prevalence of ED is high among Chinese diabetic men. After adjusting for age, diabetic duration and medication, atherosclerotic risks are still dominant in diabetic ED in the absence of clinical cardiovascular symptoms. A higher carotid IMT and the presence of lower limb plaques indicate a tight correlation between peripheral atherosclerosis and diabetic ED. Therefore, it is of significant importance to screen ED in diabetics ahead of clinical evidence of CVDs to prevent the further development of severe symptomatic CVDs. Early detection and management of ED may help improve quality of care.

\section{Acknowledgments}

Funding: This work was supported by the grants from the National Natural Sciences Foundation of China (No. 81702518 and No.81500636), and a grant from Huazhong University of Science and Technology (No. 2019kfyXKJC06).

\section{Footnote}

Reporting Checklist: The authors have completed the STROBE reporting checklist. Available at http://dx.doi. org/10.21037/tau-20-999

Data Sharing Statement: Available at http://dx.doi. org/10.21037/tau-20-999

Conflicts of Interest: All authors have completed the ICMJE uniform disclosure form (available at http://dx.doi. org/10.21037/tau-20-999). JL serves as an unpaid editorial board member of Translational Andrology and Urology. The other authors have no conflicts of interest to declare.

Ethical Statement: The authors are accountable for all aspects of the work in ensuring that questions related to the accuracy or integrity of any part of the work are appropriately investigated and resolved. This study was conducted in accordance with the Declaration of Helsinki (as revised in 2013) and approved by Institutional Research Ethics Committee of Tongji Medical College, Huazhong University of Science and Technology (S1119). Informed consent was taken from all the patients before the study.

Open Access Statement: This is an Open Access article distributed in accordance with the Creative Commons Attribution-NonCommercial-NoDerivs 4.0 International License (CC BY-NC-ND 4.0), which permits the noncommercial replication and distribution of the article with the strict proviso that no changes or edits are made and the original work is properly cited (including links to both the formal publication through the relevant DOI and the license). See: https://creativecommons.org/licenses/by-nc-nd/4.0/. 


\section{References}

1. Cho NH, Shaw JE, Karuranga S, et al. IDF Diabetes Atlas: Global estimates of diabetes prevalence for 2017 and projections for 2045. Diabetes Res Clin Pract 2018;138:271-81.

2. Baena-Díez JM, Peñafiel J, Subirana I, et al. Risk of CauseSpecific Death in Individuals With Diabetes: A Competing Risks Analysis. Diabetes Care 2016;39:1987-95.

3. Thorve VS, Kshirsagar AD, Vyawahare NS, et al. Diabetes-induced erectile dysfunction: epidemiology, pathophysiology and management. J Diabetes Complications 2011;25:129-36.

4. Derosa G, Romano D, Tinelli C, et al. Prevalence and associations of erectile dysfunction in a sample of Italian males with type 2 diabetes. Diabetes Res Clin Pract 2015;108:329-35.

5. Gandaglia G, Briganti A, Jackson G, et al. A systematic review of the association between erectile dysfunction and cardiovascular disease. Eur Urol 2014;65:968-78.

6. Chew KK, Finn J, Stuckey B, et al. Erectile dysfunction as a predictor for subsequent atherosclerotic cardiovascular events: findings from a linked-data study. J Sex Med 2010;7:192-202.

7. Lahoz C, Mostaza JM, Salinero-Fort MA, et al. Peripheral Atherosclerosis in Patients With Erectile Dysfunction: A Population-Based Study. J Sex Med 2016;13:63-9.

8. Katakami N. Mechanism of Development of Atherosclerosis and Cardiovascular Disease in Diabetes Mellitus. J Atheroscler Thromb 2018;25:27-39.

9. Stuckey BG, Walsh JP, Ching HL, et al. Erectile dysfunction predicts generalised cardiovascular disease: evidence from a case-control study. Atherosclerosis 2007;194:458-64.

10. Chiurlia E, D'Amico R, Ratti C, et al. Subclinical coronary artery atherosclerosis in patients with erectile dysfunction. J Am Coll Cardiol 2005;46:1503-6.

11. Kirby M, Jackson G, Simonsen U. Endothelial dysfunction links erectile dysfunction to heart disease. Int J Clin Pract 2005;59:225-9.

12. Bocchio $M$, Scarpelli $P$, Necozione $S$, et al. Intima-media thickening of common carotid arteries is a risk factor for severe erectile dysfunction in men with vascular risk factors but no clinical evidence of atherosclerosis. J Urol 2005;173:526-9.

13. Vlachopoulos C, Aznaouridis K, Ioakeimidis N, et al. Arterial function and intima-media thickness in hypertensive patients with erectile dysfunction. J
Hypertens 2008;26:1829-36.

14. Severo MD, Leiria LF, Ledur Pdos S, et al. Association between erectile dysfunction and echocardiographic variables of ventricular hypertrophy and diastolic function in hypertensive patients with type 2 diabetes mellitus: a cross-sectional study. J Diabetes 2014;6:586-94.

15. Anderson JL, Halperin JL, Albert NM, et al. Management of patients with peripheral artery disease (compilation of 2005 and 2011 ACCF/AHA guideline recommendations): a report of the American College of Cardiology Foundation/American Heart Association Task Force on Practice Guidelines. Circulation 2013;127:1425-43.

16. Utomo E, Blok BF, Pastoor H, et al. The measurement properties of the five-item International Index of Erectile Function (IIEF-5): a Dutch validation study. Andrology 2015;3:1154-9.

17. Zhang FL, Xing YQ, Guo ZN, et al. Prevalence and risk factors for diabetes and impaired fasting glucose in Northeast China: Results from the 2016 China National Stroke Screening Survey. Diabetes Res Clin Pract 2018;144:302-13.

18. Aboyans V, Criqui MH, Abraham P, et al. Measurement and interpretation of the ankle-brachial index: a scientific statement from the American Heart Association. Circulation 2012;126:2890-909.

19. Yu LW, Kong AP, Tong PC, et al. Evaluation of erectile dysfunction and associated cardiovascular risk using structured questionnaires in Chinese type 2 diabetic men. Int J Androl 2010;33:853-60.

20. Chen S, Peng D, Xu X, et al. Assessment of erectile dysfunction and associated psychological distress in Chinese men with type 2 diabetes mellitus. Int J Impot Res 2017;29:210-4.

21. Bloomgarden ZT. Endothelial dysfunction, neuropathy and the diabetic foot, diabetic mastopathy and erectile dysfunction. Diabetes Care 1998;21:183-9.

22. Gandaglia G, Briganti A, Montorsi P, et al. Diagnostic and Therapeutic Implications of Erectile Dysfunction in Patients with Cardiovascular Disease. Eur Urol 2016;70:219-22.

23. Keenan HA. Do erectile dysfunction and cardiovascular disease have the same mechanism? Eur Urol 2014;65:979-80.

24. Montorsi P, Ravagnani PM, Galli S, et al. The artery size hypothesis: a macrovascular link between erectile dysfunction and coronary artery disease. Am J Cardiol 2005;96:19M-23M.

25. Chambless LE, Folsom AR, Davis V, et al. Risk Factors 
for Progression of Common Carotid Atherosclerosis: The Atherosclerosis Risk in Communities Study, 1987-1998. Am J Epidemiol 2002;155:38-47.

26. Pelit ES, Dokumaci DS, Kati B, et al. Carotid artery intima-media thickness can predict the response of patients with erectile dysfunction to phosphodiesterase 5 inhibitors. Int J Impot Res 2019;31:139-44.

27. Caretta N, Palego P, Ferlin A, et al. Resumption of spontaneous erections in selected patients affected by erectile dysfunction and various degrees of carotid wall alteration: role of tadalafil. Eur Urol 2005;48:326-31; discussion 331-2.

28. Nezu T, Hosomi N, Aoki S, et al. Carotid Intima-Media Thickness for Atherosclerosis. J Atheroscler Thromb 2016;23:18-31.

29. Yao FJ, Zhang YD, Wan Z, et al. Erectile dysfunction is associated with subclinical carotid vascular disease in young men lacking widely-known risk factors. Asian J Androl 2018;20:400-4.

Cite this article as: Yuan P, Ma D, Zhang Y, Gao X, Wang J, Li R, Liu Z, Wang T, Yang Y, Yuan G, Yu X, Wang S, Liu J, Liu X. Analysis of cardiovascular risks for erectile dysfunction in Chinese patients with type 2 diabetes mellitus lacking clinical symptoms of cardiovascular diseases. Transl Androl Urol 2020;9(6):2500-2509. doi: 10.21037/tau-20-999
30. Plichart M, Celermajer DS, Zureik M, et al. Carotid intima-media thickness in plaque-free site, carotid plaques and coronary heart disease risk prediction in older adults. The Three-City Study. Atherosclerosis 2011;219:917-24.

31. Lee JH, Kim SK, Lee DG. Associations of carotid artery plaque with lower urinary tract symptoms and erectile dysfunction. Int Urol Nephrol 2014;46:2263-70.

32. Goksu C, Deveer M, Sivrioglu AK, et al. Peripheral atherosclerosis in patients with arterial erectile dysfunction. Int J Impot Res 2014;26:55-60.

33. Li L, Yu H, Zhu J, et al. The combination of carotid and lower extremity ultrasonography increases the detection of atherosclerosis in type 2 diabetes patients. J Diabetes Complications 2012;26:23-8.

34. Foresta C, Palego P, Schipilliti M, et al. Asymmetric development of peripheral atherosclerosis in patients with erectile dysfunction: an ultrasonographic study. Atherosclerosis 2008;197:889-95. 